J. T. Cho, K. Sekigawa and L. Vanhecke

Nagoya Math. J.

Vol. 146 (1997), 13-29

\title{
VOLUME-PRESERVING GEODESIC SYMMETRIES ON FOUR-DIMENSIONAL HERMITIAN EINSTEIN SPACES
}

\author{
J. T. CHO, K. SEKIGAWA AND L. VANHECKE
}

\begin{abstract}
We prove that a four-dimensional Hermitian Einstein space is weakly $*$-Einsteinian and use this result to show that all geodesic symmetries are volume-preserving (up to sign) if and only if it is local symmetric.
\end{abstract}

\section{$\S 1$. Introduction}

Riemannian manifolds such that all (local) geodesic symmetries are volume-preserving (up to sign) or equivalently, are divergence-preserving, have been introduced in [5] and are called D'Atri spaces [27]. The first examples which are not locally symmetric were discovered in [4], [6]. These are the naturally reductive homogeneous spaces. Since then, many other classes of examples has been found and studied. The main classes are the following: Riemannian g.o. spaces (i.e., spaces such that every geodesic is an orbit of a one-parameter group of isometries), commutative spaces (i.e., homogeneous spaces whose algebra of all differential operators which are invariant under all isometries is commutative), generalized Heisenberg groups, harmonic spaces (in particular, the Damek-Ricci examples), weakly symmetric spaces, $\mathcal{S C}$-spaces (i.e., spaces such that the principal curvatures of small geodesic spheres have antipodal symmetry), probabilistic commutative spaces, $\mathcal{T C}$ - and $\mathcal{C}_{0}$-spaces (see [1], [2] for more details). Of course, any manifold which is locally isometric to one of these examples is also a D'Atri space. We refer to [1], [2], [12], [26], [28] for more information and further references, in particular to the papers where these spaces have been introduced and to the extensive survey paper [13].

It is worthwhile to note that a D'Atri space is always analytic in normal coordinates [10], [22]. Moreover, their classification is completely known for dimensions smaller than four [12] but for higher dimensions the problem is completely open. Further, to our knowledge, an example which is not locally

Received June 6, 1995. 
homogeneous is not known yet and this led to the following

Problem 1. Are D'Atri spaces necessarily locally homogeneous?

In the framework of four-dimensional geometry some partial results are known. It is proved in [20], [21] that four-dimensional Kähler or 2-stein D'Atri spaces are locally symmetric. In view of Problem 1 and Jensen's theorem stating that locally homogeneous four-dimensional Einstein spaces are locally symmetric, the following problem arises naturally:

Problem 2. Is any (connected) four-dimensional Einstein D'Atri space locally symmetric?

The main purpose of this paper is to provide a positive answer for the class of Hermitian spaces:

THEOREM A. A connected four-dimensional Hermitian Einstein space with volume-preserving geodesic symmetries is locally symmetric.

Note that the theorem does not hold if the Einstein condition is deleted since the Riemannian product of the three-dimensional Heisenberg group with a line is a Hermitian D'Atri space which is not locally symmetric (see [25]).

In the course of the proof of Theorem A we will also show the following

THEOREM B. A four-dimensional Hermitian Einstein manifold is weakly $*$-Einsteinian.

See Section 2 for the definition of this last notion.

\section{$\S 2$. Proof of Theorem B}

Let $M=(M, J, g)$ be a (connected) $2 n$-dimensional almost Hermitian manifold. We denote by $\nabla, R, \rho$ and $\tau$ the Levi Civita connection, Riemannian curvature tensor, Ricci tensor and scalar curvature, respectively. For $R$ we take the sign convention

$$
R(X, Y)=\nabla_{[X, Y]}-\left[\nabla_{X}, \nabla_{Y}\right]
$$

for $X, Y \in \mathcal{X}(M)$ where $\mathcal{X}(M)$ denotes the Lie algebra of all smooth vector fields on $M$. Further we denote by $\rho^{*}$ the Ricci $*$-tensor defined by

$$
\rho^{*}(x, y)=-\frac{1}{2} \operatorname{trace}(z \mapsto R(x, J y) J z)
$$


for $x, y, z \in T_{m} M$, the tangent space at $m \in M$. The $*$-scalar curvature is given by

$$
\tau^{*}=\operatorname{trace} Q^{*}
$$

where $Q^{*}$ is the endomorphism field defined by $g\left(Q^{*} x, y\right)=\rho^{*}(x, y)$. Note that (2.2) implies at once that $\rho^{*}(x, y)=\rho^{*}(J y, J x)$ for all $x, y \in T_{m} M$. For a Kähler manifold the Ricci *-tensor coincides with the Ricci tensor.

An almost Hermitian manifold $M$ is said to be a weakly $*$-Einstein space if $\rho^{*}=\lambda^{*} g$ for some smooth function $\lambda^{*}$ on $M$. In this case $\tau^{*}=2 \lambda^{*} n$. $M$ is said to be a $*$-Einstein space if $\lambda^{*}$, or equivalently, $\tau^{*}$ is constant. We note that these notions appear naturally in the study of non-Kählerian metrics. For example, in [15] D. Page constructed a non-Kählerian Hermitian Einstein metric on $\mathbb{C P}^{2}$ with one point blown up and it was shown in [11] that with this metric the Hermitian surface is weakly $*$-Einsteinian with positive $*$-scalar curvature (see also Theorem 2.1 and the note following it). Theorem B shows that any four-dimensional Hermitian Einstein space is always weakly $*$-Einsteinian. We also refer to [24] where it is shown how $\rho^{*}$ naturally appears in the decomposition of the space of curvature tensors on an almost Hermitian manifold.

Next, we assume that $M=(M, J, g)$ is a four-dimensional Hermitian manifold. Then we have

$$
\begin{aligned}
2 g\left(\left(\nabla_{X} J\right) Y, Z\right)=\omega(J Y) g(X, Z) & +\omega(Y) g(X, J Z) \\
& -\omega(J Z) g(X, Y)-\omega(Z) g(X, J Y)
\end{aligned}
$$

for $X, Y, Z \in \mathcal{X}(M)$ and where $\omega$ is the Lee form of $M$ given by $\omega=\delta \Omega \circ J$, $\Omega$ being the Kähler form on $M$ defined by $\Omega(X, Y)=g(X, J Y)$ for $X$, $Y \in \mathcal{X}(M)[18]$. Further, let $\left\{e_{i}, i=1, \cdots, 4\right\}=\left\{e_{1}, e_{2}=J e_{1}, e_{3}, e_{4}=\right.$ $\left.J e_{3}\right\}$ be an orthonormal basis of $T_{m} M$. We adopt the following notational convention:

$$
\begin{aligned}
J_{i j}= & g\left(J e_{i}, e_{j}\right), \quad \nabla_{i} J_{j k}=g\left(\left(\nabla_{e_{i}} J\right) e_{j}, e_{k}\right), \\
\nabla_{\bar{i}} J_{j k}= & g\left(\left(\nabla_{J e_{i}} J\right) e_{j}, e_{k}\right), \cdots, \nabla_{\bar{i}} J_{\bar{j} \bar{k}}=g\left(\left(\nabla_{J e_{i}} J\right) J e_{j}, J e_{k}\right)=-\nabla_{\bar{i}} J_{j k}, \\
\rho_{i j}= & \rho\left(e_{i}, e_{j}\right), \rho_{\bar{i} j}=\rho\left(J e_{i}, e_{j}\right), \rho_{\bar{i} \bar{j}}=\rho\left(J e_{i}, J e_{j}\right), \\
R_{i j k \ell}= & g\left(R\left(e_{i}, e_{j}\right) e_{k}, e_{\ell}\right), \quad R_{\overline{i j k \ell}}=g\left(R\left(J e_{i}, e_{j}\right) e_{k}, e_{\ell}\right), \cdots, \\
& R_{\bar{i} \bar{j} \bar{k} \bar{\ell}}=g\left(R\left(J e_{i}, J e_{j}\right) J e_{k}, J e_{\ell}\right),
\end{aligned}
$$


etc., $1 \leq i, j \leq 4$. Then, by using (2.1) and (2.4), we get

$$
\begin{array}{ll}
-2( & \left.R_{i j \bar{k} \ell}+R_{i j k \bar{\ell}}\right)=\delta_{j \ell}\left(\nabla_{i} \omega_{\bar{k}}+\frac{1}{2} \omega_{\bar{k}} \omega_{i}-\frac{1}{2}\|\omega\|^{2} J_{k i}\right) \\
& -\delta_{i \ell}\left(\nabla_{j} \omega_{\bar{k}}+\frac{1}{2} \omega_{\bar{k}} \omega_{j}-\frac{1}{2}\|\omega\|^{2} J_{k j}\right)-\delta_{j k}\left(\nabla_{i} \omega_{\bar{\ell}}+\frac{1}{2} \omega_{\bar{\ell}} \omega_{i}-\frac{1}{2}\|\omega\|^{2} J_{\ell i}\right) \\
& +\delta_{i k}\left(\nabla_{j} \omega_{\bar{\ell}}+\frac{1}{2} \omega_{\bar{\ell}} \omega_{j}-\frac{1}{2}\|\omega\|^{2} J_{\ell j}\right)+J_{\ell j}\left(\nabla_{i} \omega_{k}+\frac{1}{2} \omega_{i} \omega_{k}\right) \\
& -J_{\ell i}\left(\nabla_{j} \omega_{k}+\frac{1}{2} \omega_{j} \omega_{k}\right)-J_{k j}\left(\nabla_{i} \omega_{\ell}+\frac{1}{2} \omega_{i} \omega_{\ell}\right)+J_{k i}\left(\nabla_{j} \omega_{\ell}+\frac{1}{2} \omega_{j} \omega_{\ell}\right)
\end{array}
$$

(see [18]). From this we obtain

$$
\tau-\tau^{*}=2 \delta \omega+\|\omega\|^{2}
$$

From now on we suppose that $M$ is also Einsteinian. First we shall provide a proof of Theorem B and derive the relation (2.50) which will be needed to pove Theorem A.

We start by giving a series of relations which follow at once from (2.6) and (2.7):

$$
\begin{aligned}
& 2\left(R_{1313}-R_{1324}\right)=-\nabla_{1} \omega_{1}-\nabla_{3} \omega_{3}-\frac{1}{2} \omega_{1}^{2}-\frac{1}{2} \omega_{3}^{2}+\frac{1}{2}\|\omega\|^{2} \\
& 2\left(R_{2413}-R_{2424}\right)=\nabla_{2} \omega_{2}+\nabla_{4} \omega_{4}+\frac{1}{2} \omega_{2}^{2}+\frac{1}{2} \omega_{4}^{2}-\frac{1}{2}\|\omega\|^{2} \\
& 2\left(R_{1423}+R_{1414}\right)=-\nabla_{1} \omega_{1}-\nabla_{4} \omega_{4}-\frac{1}{2} \omega_{1}^{2}-\frac{1}{2} \omega_{4}^{2}+\frac{1}{2}\|\omega\|^{2} \\
& 2\left(R_{2323}+R_{2314}\right)=-\nabla_{2} \omega_{2}-\nabla_{3} \omega_{3}-\frac{1}{2} \omega_{2}^{2}-\frac{1}{2} \omega_{3}^{2}+\frac{1}{2}\|\omega\|^{2}
\end{aligned}
$$

Then (2.8) and (2.9) yield

$$
\begin{gathered}
2\left(R_{1313}-R_{2424}\right)=-\nabla_{1} \omega_{1}-\nabla_{3} \omega_{3}+\nabla_{2} \omega_{2}+\nabla_{4} \omega_{4} \\
-\frac{1}{2}\left(\omega_{1}^{2}+\omega_{3}^{2}-\omega_{2}^{2}-\omega_{4}^{2}\right), \\
2\left(R_{1313}+R_{2424}-2 R_{1324}\right)=\delta \omega+\frac{1}{2}\|\omega\|^{2}=\frac{1}{2}\left(\tau-\tau^{*}\right) .
\end{gathered}
$$

Similarly, from (2.10) and (2.11) we have

$$
\begin{aligned}
2\left(R_{2323}-R_{1414}\right)=\nabla_{1} \omega_{1}+\nabla_{4} \omega_{4}-\nabla_{2} \omega_{2}-\nabla_{3} \omega_{3} & \\
& +\frac{1}{2}\left(\omega_{1}^{2}+\omega_{4}^{2}-\omega_{2}^{2}-\omega_{3}^{2}\right),
\end{aligned}
$$




$$
2\left(R_{1414}+R_{2323}+2 R_{1423}\right)=\delta \omega+\frac{1}{2}\|\omega\|^{2}=\frac{1}{2}\left(\tau-\tau^{*}\right) .
$$

Finally, from (2.8) and (2.10) we derive

$$
\begin{aligned}
R_{1234}=- & R_{1342}-R_{1423} \\
R_{1313}+R_{1414} & +\frac{1}{2}\left(\nabla_{1} \omega_{1}+\nabla_{3} \omega_{3}+\frac{1}{2} \omega_{1}^{2}+\frac{1}{2} \omega_{3}^{2}-\frac{1}{2}\|\omega\|^{2}\right) \\
& +\frac{1}{2}\left(\nabla_{1} \omega_{1}+\nabla_{4} \omega_{4}+\frac{1}{2} \omega_{1}^{2}+\frac{1}{2} \omega_{4}^{2}-\frac{1}{2}\|\omega\|^{2}\right) \\
6)=\frac{\tau}{4}-R_{1212}+ & \frac{1}{2}\left(-\delta \omega+\frac{1}{2}\|\omega\|^{2}\right) \\
& +\frac{1}{2}\left(\nabla_{1} \omega_{1}-\nabla_{2} \omega_{2}+\frac{1}{2} \omega_{1}^{2}-\frac{1}{2} \omega_{2}^{2}\right)-\frac{1}{2}\|\omega\|^{2} \\
= & -R_{1212}+\frac{\tau^{*}}{4}+\frac{1}{2}\left(\nabla_{1} \omega_{1}-\nabla_{2} \omega_{2}+\frac{1}{2} \omega_{1}^{2}-\frac{1}{2} \omega_{2}^{2}\right) .
\end{aligned}
$$

Again, by (2.6) we get

$$
\begin{aligned}
& 2\left(R_{1213}-R_{1224}\right)=-\nabla_{1} \omega_{4}-\nabla_{2} \omega_{3}-\frac{1}{2} \omega_{1} \omega_{4}-\frac{1}{2} \omega_{2} \omega_{3}, \\
& 2\left(R_{3413}-R_{3424}\right)=\nabla_{3} \omega_{2}+\nabla_{4} \omega_{1}+\frac{1}{2} \omega_{2} \omega_{3}+\frac{1}{2} \omega_{1} \omega_{4}, \\
& 2\left(R_{1214}+R_{1223}\right)=\nabla_{1} \omega_{3}-\nabla_{2} \omega_{4}+\frac{1}{2} \omega_{1} \omega_{3}-\frac{1}{2} \omega_{2} \omega_{4}, \\
& 2\left(R_{3414}+R_{3423}\right)=\nabla_{4} \omega_{2}-\nabla_{3} \omega_{1}+\frac{1}{2} \omega_{2} \omega_{4}-\frac{1}{2} \omega_{1} \omega_{3}, \\
& 2\left(R_{1314}+R_{1323}\right)=-\nabla_{1} \omega_{2}-\nabla_{3} \omega_{4}-\frac{1}{2} \omega_{1} \omega_{2}-\frac{1}{2} \omega_{3} \omega_{4}, \\
& 2\left(R_{2423}+R_{2414}\right)=-\nabla_{2} \omega_{1}-\nabla_{4} \omega_{3}-\frac{1}{2} \omega_{1} \omega_{2}-\frac{1}{2} \omega_{3} \omega_{4} .
\end{aligned}
$$

Using the Einstein condition, the relations (2.17) - (2.22) yield

$$
\begin{aligned}
& R_{1242}^{2}=R_{1213}^{2}+R_{1213}(\left.\nabla_{2} \omega_{3}+\nabla_{1} \omega_{4}+\frac{1}{2} \omega_{2} \omega_{3}+\frac{1}{2} \omega_{1} \omega_{4}\right) \\
&+ \frac{1}{4}\left(\nabla_{2} \omega_{3}+\nabla_{1} \omega_{4}+\frac{1}{2} \omega_{2} \omega_{3}+\frac{1}{2} \omega_{1} \omega_{4}\right)^{2}, \\
& R_{1343}^{2}=R_{1213}^{2}-R_{1213}\left(\nabla_{3} \omega_{2}+\nabla_{4} \omega_{1}+\frac{1}{2} \omega_{2} \omega_{3}+\frac{1}{2} \omega_{1} \omega_{4}\right) \\
&+\frac{1}{4}\left(\nabla_{3} \omega_{2}+\nabla_{4} \omega_{1}+\frac{1}{2} \omega_{2} \omega_{3}+\frac{1}{2} \omega_{1} \omega_{4}\right)^{2},
\end{aligned}
$$




$$
\begin{gathered}
R_{1232}^{2}=R_{1214}^{2}+R_{1214}\left(\nabla_{2} \omega_{4}-\nabla_{1} \omega_{3}-\frac{1}{2} \omega_{1} \omega_{3}+\frac{1}{2} \omega_{2} \omega_{4}\right) \\
+\frac{1}{4}\left(\nabla_{2} \omega_{4}-\nabla_{1} \omega_{3}-\frac{1}{2} \omega_{1} \omega_{3}+\frac{1}{2} \omega_{2} \omega_{4}\right)^{2}, \\
R_{3414}^{2}=R_{1214}^{2}+R_{1214}\left(\nabla_{3} \omega_{1}-\nabla_{4} \omega_{2}+\frac{1}{2} \omega_{1} \omega_{3}-\frac{1}{2} \omega_{2} \omega_{4}\right) \\
+\frac{1}{4}\left(\nabla_{3} \omega_{1}-\nabla_{4} \omega_{2}+\frac{1}{2} \omega_{1} \omega_{3}-\frac{1}{2} \omega_{2} \omega_{4}\right)^{2}, \\
R_{1323}^{2}=R_{1314}^{2}+R_{1314}\left(\nabla_{1} \omega_{2}+\nabla_{3} \omega_{4}+\frac{1}{2} \omega_{1} \omega_{2}+\frac{1}{2} \omega_{3} \omega_{4}\right) \\
+\frac{1}{4}\left(\nabla_{1} \omega_{2}+\nabla_{3} \omega_{4}+\frac{1}{2} \omega_{1} \omega_{2}+\frac{1}{2} \omega_{3} \omega_{4}\right)^{2}, \\
R_{2414}^{2}=R_{1314}^{2}-R_{1314}\left(\nabla_{4} \omega_{3}+\nabla_{2} \omega_{1}+\frac{1}{2} \omega_{3} \omega_{4}+\frac{1}{2} \omega_{1} \omega_{2}\right) \\
+\frac{1}{4}\left(\nabla_{4} \omega_{3}+\nabla_{2} \omega_{1}+\frac{1}{2} \omega_{3} \omega_{4}+\frac{1}{2} \omega_{1} \omega_{2}\right)^{2} .
\end{gathered}
$$

Now, on the Hermitian manifold the relation $\sum_{i=1}^{4} \nabla_{i} \omega_{\bar{i}}=0$ holds [23], i.e., we have

$$
\nabla_{1} \omega_{2}-\nabla_{2} \omega_{1}+\nabla_{3} \omega_{4}-\nabla_{4} \omega_{3}=0
$$

Further, from (2.21), (2.22) and the Einstein condition we obtain

$$
\nabla_{1} \omega_{2}+\nabla_{2} \omega_{1}+\nabla_{3} \omega_{4}+\nabla_{4} \omega_{3}+\omega_{1} \omega_{2}+\omega_{3} \omega_{4}=0
$$

By direct calculation we have

$$
\begin{aligned}
& \left(\nabla_{1} \omega_{2}+\nabla_{3} \omega_{4}+\frac{1}{2} \omega_{1} \omega_{2}+\frac{1}{2} \omega_{3} \omega_{4}\right)^{2} \\
& +\left(\nabla_{2} \omega_{1}+\nabla_{4} \omega_{3}+\frac{1}{2} \omega_{1} \omega_{2}+\frac{1}{2} \omega_{3} \omega_{4}\right)^{2} \\
& =\left(\nabla_{1} \omega_{2}\right)^{2}+\left(\nabla_{2} \omega_{1}\right)^{2}+\left(\nabla_{3} \omega_{4}\right)^{2}+\left(\nabla_{4} \omega_{3}\right)^{2}+2\left(\nabla_{1} \omega_{2}\right) \nabla_{3} \omega_{4} \\
& +2\left(\nabla_{2} \omega_{1}\right) \nabla_{4} \omega_{3}+\omega_{1} \omega_{2} \nabla_{1} \omega_{2}+\omega_{3} \omega_{4} \nabla_{1} \omega_{2}+\omega_{1} \omega_{2} \nabla_{3} \omega_{4} \\
& +\omega_{3} \omega_{4} \nabla_{3} \omega_{4}+\omega_{1} \omega_{2} \nabla_{2} \omega_{1}+\omega_{3} \omega_{4} \nabla_{2} \omega_{1}+\omega_{1} \omega_{2} \nabla_{4} \omega_{3} \\
& +\omega_{3} \omega_{4} \nabla_{4} \omega_{3}+\frac{1}{2}\left(\omega_{1} \omega_{2}+\omega_{3} \omega_{4}\right)^{2} .
\end{aligned}
$$

Next, by (2.29) and (2.30) we get

$$
\begin{aligned}
\left(\omega_{1} \omega_{2}+\omega_{3} \omega_{4}\right)^{2}=\left(\nabla_{1} \omega_{2}+\nabla_{2} \omega_{1}+\right. & \left.\nabla_{3} \omega_{4}+\nabla_{4} \omega_{3}\right)^{2} \\
& -\left(\nabla_{1} \omega_{2}-\nabla_{2} \omega_{1}+\nabla_{3} \omega_{4}-\nabla_{4} \omega_{3}\right)^{2} \\
& \\
2.32) \quad 4\left\{\left(\nabla_{1} \omega_{2}\right) \nabla_{2} \omega_{1}+\right. & \left(\nabla_{3} \omega_{4}\right) \nabla_{4} \omega_{3} \\
& \left.+\left(\nabla_{1} \omega_{2}\right) \nabla_{4} \omega_{3}+\left(\nabla_{2} \omega_{1}\right) \nabla_{3} \omega_{4}\right\} .
\end{aligned}
$$


So, from (2.30)-(2.32) we obtain

$$
\begin{gathered}
\left(\nabla_{1} \omega_{2}+\nabla_{3} \omega_{4}+\frac{1}{2} \omega_{1} \omega_{2}+\frac{1}{2} \omega_{3} \omega_{4}\right)^{2} \\
+\left(\nabla_{2} \omega_{1}+\nabla_{4} \omega_{3}+\frac{1}{2} \omega_{1} \omega_{2}+\frac{1}{2} \omega_{3} \omega_{4}\right)^{2} \\
=\left(\nabla_{1} \omega_{2}+\nabla_{2} \omega_{1}+\nabla_{3} \omega_{4}+\nabla_{4} \omega_{3}+\omega_{1} \omega_{2}+\omega_{3} \omega_{4}\right)^{2} \\
\quad-\omega_{1} \omega_{2} \nabla_{1} \omega_{2}-\omega_{3} \omega_{4} \nabla_{1} \omega_{2}-\omega_{3} \omega_{4} \nabla_{3} \omega_{4} \\
\quad-\omega_{1} \omega_{2} \nabla_{3} \omega_{4}-\omega_{1} \omega_{2} \nabla_{2} \omega_{1}-\omega_{3} \omega_{4} \nabla_{2} \omega_{1} \\
\quad-\omega_{1} \omega_{2} \nabla_{4} \omega_{3}-\omega_{3} \omega_{4} \nabla_{4} \omega_{3}-\left(\omega_{1} \omega_{2}+\omega_{3} \omega_{4}\right)^{2} \\
=-\left(\nabla_{1} \omega_{2}+\nabla_{2} \omega_{1}+\nabla_{3} \omega_{4}+\nabla_{4} \omega_{3}\right. \\
\left.\quad+\omega_{1} \omega_{2}+\omega_{3} \omega_{4}\right)\left(\omega_{1} \omega_{2}+\omega_{3} \omega_{4}\right) \\
=0
\end{gathered}
$$

Hence, we have

$$
\begin{aligned}
& \nabla_{1} \omega_{2}+\nabla_{3} \omega_{4}+\frac{1}{2} \omega_{1} \omega_{2}+\frac{1}{2} \omega_{3} \omega_{4}=0 \\
& \nabla_{2} \omega_{1}+\nabla_{4} \omega_{3}+\frac{1}{2} \omega_{1} \omega_{2}+\frac{1}{2} \omega_{3} \omega_{4}=0
\end{aligned}
$$

Since the basis $\left(e_{1}, e_{2}=J e_{1}, e_{3}, e_{4}=J e_{3}\right)$ is chosen arbitrarily, we may replace $\left(e_{1}, e_{2}\right)$ by $\left(e_{2},-e_{1}\right)$ in $(2.33)$. Then we also get

$$
\begin{aligned}
& -\nabla_{2} \omega_{1}+\nabla_{3} \omega_{4}-\frac{1}{2} \omega_{1} \omega_{2}+\frac{1}{2} \omega_{3} \omega_{4}=0 \\
& -\nabla_{1} \omega_{2}+\nabla_{4} \omega_{3}-\frac{1}{2} \omega_{1} \omega_{2}+\frac{1}{2} \omega_{3} \omega_{4}=0 .
\end{aligned}
$$

So, (2.33) and (2.34) yield

$$
\begin{aligned}
& \nabla_{1} \omega_{2}+\nabla_{2} \omega_{1}+\omega_{1} \omega_{2}=0 \\
& \nabla_{3} \omega_{4}+\nabla_{4} \omega_{3}+\omega_{3} \omega_{4}=0 .
\end{aligned}
$$

Further, using the Einstein condition, we obtain

$$
\begin{aligned}
R_{3434} & =\frac{\tau}{4}-\left(R_{1414}+R_{2424}\right) \\
& =\frac{\tau}{4}-\left(\frac{\tau}{4}-R_{1212}-R_{1313}+\frac{\tau}{4}-R_{2121}-R_{2323}\right) \\
& =-\frac{\tau}{4}+2 R_{1212}+R_{1313}+R_{2323}
\end{aligned}
$$




$$
\begin{aligned}
& =-\frac{\tau}{4}+2 R_{1212}+\frac{\tau}{4}-R_{4343} \\
& =2 R_{1212}-R_{4343}
\end{aligned}
$$

and hence,

$$
R_{1212}=R_{3434}
$$

From this we get

$$
\begin{aligned}
& R_{1313} \\
& =\frac{\tau}{4}-R_{2323}-R_{4343}=\frac{\tau}{4}-R_{2323}-R_{2121}=\frac{\tau}{4}-\left(\frac{\tau}{4}-R_{2424}\right)=R_{2424}
\end{aligned}
$$

So, we have

$$
R_{1313}=R_{2424}
$$

Similarly, we obtain

$$
R_{1414}=R_{2323}
$$

Thus, by (2.12), (2.14), (2.37) and (2.38) we get

$$
\begin{aligned}
& \nabla_{1} \omega_{1}-\nabla_{2} \omega_{2}+\frac{1}{2} \omega_{1}^{2}-\frac{1}{2} \omega_{2}^{2}=0 \\
& \nabla_{3} \omega_{3}-\nabla_{4} \omega_{4}+\frac{1}{2} \omega_{3}^{2}-\frac{1}{2} \omega_{4}^{2}=0
\end{aligned}
$$

Finally, we compute the square of the length of $R$ by making use of the previous formulas. We have

$$
\begin{aligned}
\|R\|^{2}=4 \sum_{a, b} & \left(R_{1 a 1 b}^{2}+R_{2 a 2 b}^{2}+R_{3 a 3 b}^{2}+R_{4 a 4 b}^{2}\right) \\
& -4\left(R_{1212}^{2}+R_{1313}^{2}+R_{1414}^{2}+R_{2323}^{2}+R_{2424}^{2}+R_{3434}^{2}\right) \\
& +8\left(R_{1234}^{2}+R_{1324}^{2}+R_{1423}^{2}\right) .
\end{aligned}
$$


Using the Einstein condition and the formulas (2.8)-(2.38) we first obtain

$$
\begin{aligned}
4 \sum_{a, b}\left(R_{1 a 1 b}^{2}+\right. & \left.R_{2 a 2 b}^{2}+R_{3 a 3 b}^{2}+R_{4 a 4 b}^{2}\right) \\
=4\{ & 4 \sum_{a, b} R_{1 a 1 b}^{2}+2 R_{1213}\left(\nabla_{2} \omega_{3}-\nabla_{3} \omega_{2}+\nabla_{1} \omega_{4}-\nabla_{4} \omega_{1}\right) \\
& +2 R_{1214}\left(\nabla_{2} \omega_{4}-\nabla_{4} \omega_{2}+\nabla_{3} \omega_{1}-\nabla_{1} \omega_{3}\right) \\
& +2 R_{1314}\left(\nabla_{1} \omega_{2}-\nabla_{2} \omega_{1}+\nabla_{3} \omega_{4}-\nabla_{4} \omega_{3}\right)+\frac{1}{2}\left(\nabla_{2} \omega_{3}+\nabla_{1} \omega_{4}\right. \\
& \left.+\frac{1}{2} \omega_{2} \omega_{3}+\frac{1}{2} \omega_{1} \omega_{4}\right)^{2}+\frac{1}{2}\left(\nabla_{3} \omega_{2}+\nabla_{4} \omega_{1}+\frac{1}{2} \omega_{2} \omega_{3}+\frac{1}{2} \omega_{1} \omega_{4}\right)^{2} \\
& +\frac{1}{2}\left(\nabla_{2} \omega_{4}-\nabla_{1} \omega_{3}+\frac{1}{2} \omega_{2} \omega_{4}-\frac{1}{2} \omega_{1} \omega_{3}\right)^{2}+\frac{1}{2}\left(\nabla_{4} \omega_{2}-\nabla_{3} \omega_{1}\right. \\
& \left.+\frac{1}{2} \omega_{2} \omega_{4}-\frac{1}{2} \omega_{1} \omega_{3}\right)^{2} .
\end{aligned}
$$

So, using (2.29) and (2.39), we see that (2.41) reduces to

$$
\begin{aligned}
4 \sum_{a, b} & \left(R_{1 a 1 b}^{2}+R_{2 a 2 b}^{2}+R_{3 a 3 b}^{2}+R_{4 a 4 b}^{2}\right) \\
= & 16 \sum_{a, b} R_{1 a 1 b}^{2}+4 \sum_{a, b} R_{1 a 1 b}\left(\nabla_{a} \omega_{b}-\nabla_{b} \omega_{a}-\nabla_{\bar{a}} \omega_{\bar{b}}+\nabla_{\bar{b}} \omega_{\bar{a}}\right) \\
& +\sum_{a, b}\left(\nabla_{a} \omega_{b}-\nabla_{\bar{a}} \omega_{\bar{b}}+\frac{1}{2} \omega_{a} \omega_{b}-\frac{1}{2} \omega_{\bar{a}} \omega_{\bar{b}}\right)^{2} \\
= & 16 \sum_{a, b} R_{1 a 1 b}^{2}+\sum_{a, b}\left(\nabla_{a} \omega_{b}-\nabla_{\bar{a}} \omega_{\bar{b}}+\frac{1}{2} \omega_{a} \omega_{b}-\frac{1}{2} \omega_{\bar{a}} \omega_{\bar{b}}\right)^{2} .
\end{aligned}
$$

Next, from (2.13), (2.15), (2.16), (2.37) and (2.38) we have

$$
\begin{aligned}
& R_{1324}=R_{1313}-\frac{1}{8}\left(\tau-\tau^{*}\right), \\
& R_{1423}=-R_{1414}+\frac{1}{8}\left(\tau-\tau^{*}\right), \\
& R_{1234}=\frac{\tau^{*}}{4}-R_{1212}
\end{aligned}
$$

and hence,

$$
\begin{aligned}
& 8\left(R_{1234}^{2}+R_{1324}^{2}+\right.\left.R_{1423}^{2}\right)=8\left(R_{1212}^{2}+R_{1313}^{2}+R_{1414}^{2}\right) \\
&+2\left(\tau-3 \tau^{*}\right) R_{1212}+\frac{\tau^{2}}{2}-\frac{3}{4}\left(\tau-\tau^{*}\right)\left(\tau+\tau^{*}\right) .
\end{aligned}
$$


Thus, using (2.42) and $(2.44),(2.40)$ reduces to

$$
\begin{array}{r}
\|R\|^{2}=16 \sum_{a, b} R_{1 a 1 b}^{2}+\frac{\tau^{2}}{2}-\frac{3}{4}\left(\tau-\tau^{*}\right)\left(\tau+\tau^{*}\right)+2\left(\tau-3 \tau^{*}\right) R_{1212} \\
+\sum_{a, b}\left(\nabla_{a} \omega_{b}-\nabla_{\bar{a}} \omega_{\bar{b}}+\frac{1}{2} \omega_{a} \omega_{b}-\frac{1}{2} \omega_{\bar{a}} \omega_{\bar{b}}\right)^{2}
\end{array}
$$

Now we are ready to give the

Proof of Theorem B. Let $m$ be an arbitrary point of $M$ and $\left(e_{1}, e_{2}=\right.$ $\left.J e_{1}, e_{3}, e_{4}=J e_{3}\right)$ an orthonormal basis of $T_{m} M$. Further, let $G$ denote the smooth function on $M$ defined by

$$
G(m)=\sum_{a, b}\left(\nabla_{a} \omega_{b}-\nabla_{\bar{a}} \omega_{\bar{b}}+\frac{1}{2} \omega_{a} \omega_{b}-\frac{1}{2} \omega_{\bar{a}} \omega_{\bar{b}}\right)^{2}
$$

Next, put $\xi=e_{1}$ and

$$
F(\xi)=\sum_{a, b} R_{\xi a \xi b}^{2}=\sum R_{1 a 1 b}^{2}
$$

Then (2.45) may be written in the form

$$
\|R\|^{2}=16 F(\xi)+2\left(\tau-3 \tau^{*}\right) H(\xi)-\frac{1}{4}\left(\tau^{2}-3 \tau^{* 2}\right)+G
$$

at each $m \in M$, where $H(\xi)$ denotes the holomorphic sectional curvature of the holomorphic plane determined by $\xi$. Thus, for an arbitrary, not necessarily unit vector $x \in T_{m} M$, we get

$$
\begin{aligned}
\|R\|^{2} g(x, x) g(x, x)=16 F(x) & +2\left(\tau-3 \tau^{*}\right) H(x) \\
+ & \left\{\frac{1}{4}\left(3 \tau^{* 2}-\tau^{2}\right)+G\right\} g(x, x) g(x, x),
\end{aligned}
$$

where $F(x)=\sum x_{i} x_{j} x_{k} x_{\ell} R_{i a j b} R_{k a \ell b}$ and $H(x)=\sum x_{i} x_{j} x_{k} x_{\ell} R_{i \bar{j} k \bar{\ell}}$ for $x=$ $\sum x_{i} e_{i}$.

Now, by taking twice the Euclidean Laplacian $D$ in $T_{m} M$ of both members in (2.47) (or by integrating (2.46) over the unit sphere in $T_{m} M$ ) we eventually obtain, omitting the detailed computation,

$$
G(m)=0
$$


for each $m \in M$. Hence, the definition of $G$ and (2.17) - (2.22), (2.33) yield

$$
\begin{aligned}
& R_{1213}=R_{1224}, R_{1214}=-R_{1223}, R_{1314}=-R_{1323} \\
& R_{1334}=R_{2434}=-R_{1213} \\
& R_{1434}=-R_{2334}=-R_{1214} \\
& R_{1424}=-R_{2324}=R_{1314} .
\end{aligned}
$$

From this and (2.2) we get at once

$$
\rho_{1 \overline{1}}^{*}=\rho_{2 \overline{2}}^{*}=\rho_{3 \overline{3}}^{*}=\rho_{4 \overline{4}}^{*}=0
$$

and an easy computation also yields

$$
\rho_{13}^{*}=\rho_{14}^{*}=\rho_{23}^{*}=\rho_{24}^{*}=0 .
$$

Furthermore, from (2.2) and (2.36) we get

$$
\rho_{11}^{*}=\rho_{33}^{*}=R_{1212}+R_{1234} .
$$

From this we may conclude that $4 \rho^{*}=\tau^{*} g$ at each $m \in M$, and this completes the proof.

Remarks. A. From (2.47) and (2.48) we have

$$
\begin{aligned}
\|R\|^{2} g(x, x) g(x, x)=16 F(x)+2(\tau & \left.-3 \tau^{*}\right) H(x) \\
& +\frac{1}{4}\left(3 \tau^{* 2}-\tau^{2}\right) g(x, x) g(x, x)
\end{aligned}
$$

for any $x \in T_{m} M$ and all $m \in M$.

B. Using Theorem B and also Theorem 1.1 in [7], we obtain

THEOREM 2.1. Let $M$ be a four-dimensional compact Hermitian Einstein manifold. Then either

(i) $M$ is Kählerian, or

(ii) the metric of $M$ is conformal to an extremal Kähler metric with non-constant positive scalar curvature. Moreover, the scalar curvature of $M$ is a positive constant and the *-scalar curvature of $M$ is positive and non-constant.

The converse also holds. 
The proof of this theorem is given in [7] under the additional assumption that $(M, g, J)$ is weakly *-Einsteinian. Our Theorem B shows that this hypothesis is redundant.

We note here that the above result is partly contained in [14] where the author proved that a compact Hermitian Einstein surface $(M, g, J)$ is either Kählerian or there exists an extremal Kähler metric conformal to $g$ with non-positive scalar curvature. Moreover, in the last case, $(M, J)$ is obtained from $\mathbb{C P}^{2}$ by blowing up one, two, or three points in general position. We refer to [3], [15] for an example of a non-Kähler Hermitian Einstein metric on $\mathbb{C P}^{2}$ with one point blown up.

\section{$\S$ 3. Proof of Theorem A}

Let $M$ be a four-dimensional, connected, Einstein D'Atri space. Then it follows from [20], [21] that $\|R\|^{2}$ is constant on $M$. But using [9], we have more. Indeed, in [16] it is proved that a compact connected D'Atri space of arbitrary dimension is ball-homogeneous, i.e., the volume of a sufficiently small geodesic ball only depends on the radius of this ball. In [9] it is proved that this result still holds when one deletes the compactness condition. Hence, this and the formulas given in [8] imply

Proposition 3.1. Let $M$ be a four-dimensional, connected D'Atri Einstein space. Then $\|R\|^{2}$ and $\|\nabla R\|^{2}$ are constant on $M$.

In what follows we suppose that $M$ is in addition a Hermitian surface $(M, J, g)$. Let $K, L, N$ be the smooth functions on the tangent bundle $T M$ of $M$ defined by

$$
\begin{aligned}
K(x) & =x\left(\tau^{*}\right) H(x)=x\left(\tau^{*}\right) R(x, J x, x, J x), \\
L(x) & =\left(\nabla_{x} R\right)(x, J x, x, J x)-\omega(x) H(x) \\
& -\sum_{i=1}^{4} \omega\left(J e_{i}\right) R\left(x, e_{i}, x, J x\right) g(x, x), \\
N(x) & =x\left(\tau^{* 2}\right) g(x, x) g(x, x)
\end{aligned}
$$

for $x \in T_{m} M, m \in M$ and where $\left\{e_{i}, i=1,2,3,4\right\}$ is an orthonormal basis of $T_{m} M$. Further, put

$$
(\nabla R)(x)=\left(\nabla_{x} R\right)(x, J x, x, J x) .
$$

Next, let $D$ be the Euclidean Laplacian on $T_{m} M$. Then, from (2.50), (3.1), (3.2) and taking Proposition 3.1 into account, we get by a direct and 
long computation which we omit:

$$
\begin{aligned}
\left(D^{2} K\right)(x) & =16\left(\tau+3 \tau^{*}\right) x\left(\tau^{*}\right), \\
\left(D^{2} \nabla R\right)(x) & =48 x\left(\tau^{*}\right), \\
\left(D^{2} L\right)(x) & =48 x\left(\tau^{*}\right)-2\left(\tau+3 \tau^{*}\right) \omega(x), \\
\left(D^{2} N\right)(x) & =768 \tau^{*} x\left(\tau^{*}\right) .
\end{aligned}
$$

Now, since $M$ is a D'Atri-space, we have

$$
x F(x)=0
$$

for all $x \in T_{m} M$ and all $m \in M$ (see for example [20], [21]). Hence, by (2.50), (2.4), (3.1), (3.2) we obtain

$$
-24 K(x)+8\left(\tau-3 \tau^{*}\right) L(x)+3 N(x)=0 .
$$

Then, using (3.3)-(3.6), this implies

$-24\left(\tau+3 \tau^{*}\right) x\left(\tau^{*}\right)+\left(\tau-3 \tau^{*}\right)\left\{24 x\left(\tau^{*}\right)-\left(\tau+3 \tau^{*}\right) \omega(x)\right\}+144 \tau^{*} x$

and hence, we obtain

$$
\left(\tau-3 \tau^{*}\right)\left(\tau+3 \tau^{*}\right) \omega(x)=0
$$

for all $x \in T_{m} M$ and all $m \in M$.

Finally, we denote by $M_{0}, M_{1}, M_{2}$ the subsets of $M$ defined by

$$
\begin{aligned}
& M_{0}=\left\{m \in M \mid\left(\tau-3 \tau^{*}\right)\left(\tau+3 \tau^{*}\right) \neq 0 \text { at } m\right\} \\
& M_{1}=\left\{m \in M \mid \tau+3 \tau^{*}=0 \text { at } m\right\} \\
& M_{2}=\left\{m \in M \mid \tau-3 \tau^{*}=0 \text { at } m\right\} .
\end{aligned}
$$

First, we assume that $M_{0} \neq \varnothing$. Then it follows from (3.9) that the open subspace $M_{0}=\left(M_{0}, J, g\right)$ is Kählerian and hence locally symmetric [20]. Then (2.7) implies that $\tau=\tau^{*}=$ const. Therefore we may conclude that $M_{0}=\bar{M}_{0}$ and so $M_{0}=M$. Next, we assume that $M_{0}=\varnothing$. Then $M=M_{1} \cup M_{2}$. We assume now that $\tau \neq 0$. Then we have $M_{1} \cap M_{2}=\varnothing$ and hence $M_{1}=\varnothing$ or $M_{2}=\varnothing$ since $M$ is connected. First, let $M_{1}=\varnothing$ and hence $M_{2}=M$. Then (2.50) yields that $F(\xi)$ is independent of the choice of unit vector $\xi \in T_{m} M$ for all $m \in M$ and so, $M$ is a 2-stein space. It follows then from [21] that $M$ is locally symmetric. 
We are left with the case $M_{1}=M$, i.e., $\tau+3 \tau^{*}=0$ on $M$. Then, let $\left\{e_{1}, e_{2}=J e_{1}, e_{3}, e_{4}=J e_{3}\right\}$ be a unitary basis of $T_{m} M$ as constructed in the proof of Lemma 11 in [20, p. 282]. For such a basis we have

$$
R_{1213}+R_{1224}=0, \quad R_{1214}-R_{1223}=0, \quad R_{1314}=0, \quad R_{1323}=0
$$

and hence

$$
R_{3413}+R_{3424}=0, R_{3423}-R_{3414}=0, R_{2324}=0, R_{1424}=0 .
$$

Now, since $G(m)=0$, we have from $(2.17)-(2.22)$ :

$$
\begin{array}{ll}
R_{1213}-R_{1224}=0, & R_{1214}+R_{1223}=0 \\
R_{1314}+R_{1323}=0, & R_{2423}+R_{2414}=0
\end{array}
$$

and hence also

$$
R_{3413}-R_{3424}=0, \quad R_{3414}+R_{3423}=0 .
$$

So, $(3.10),\left(3.10^{\prime}\right),(3.11),\left(3.11^{\prime}\right)$ yield that this unitary basis is a SingerThorpe basis. Now, put

$$
\begin{aligned}
& a=R_{1212}=R_{3434}, \quad b=R_{1313}=R_{2424}, \quad c=R_{1414}=R_{2323}, \\
& \alpha=R_{1234}, \quad \beta=R_{1342}, \quad \gamma=R_{1423} .
\end{aligned}
$$

Then we have from $(2.43)$ and $\tau+3 \tau^{*}=0$ :

$$
\alpha=-\frac{\tau}{12}-a, \quad \beta=\frac{\tau}{6}-b, \quad \gamma=\frac{\tau}{6}-c .
$$

Next, using the expressions $[20,(7),(9)]$ for the curvature invariants $\|R\|^{2}$ and $R$, we get

$$
\begin{aligned}
& 16\left\{\left(a+\frac{\tau}{8}\right)^{2}+b^{2}+c^{2}\right\}=\frac{5}{12} \tau^{2}+\|R\|^{2}, \\
& 16\left\{4\left[\left(a+\frac{\tau}{8}\right)^{3}+b^{3}+c^{3}\right]-\tau\left[\left(a+\frac{\tau}{8}\right)^{2}+b^{2}+c^{2}\right]=\stackrel{\vee}{R}-\frac{11}{24} \tau^{3},\right.
\end{aligned}
$$

where

$$
\stackrel{\vee}{R}=-\frac{\tau^{3}}{12}+\frac{2}{3} \tau\|R\|^{2}+\frac{1}{3}\|\nabla R\|^{2}
$$


Further, we also have (since $\left.a+b+c=\frac{\tau}{4}\right)$

$$
\left(a+\frac{\tau}{8}\right)+b+c=\frac{3 \tau}{8} .
$$

So, from (3.13), (3.14), (3.16) and taking into account (3.15) and Proposition 3.1 , we see that $a, b, c$ (and hence, $\alpha, \beta, \gamma$ ) are constant. So, $M$ is curvature homogeneous and thus locally symmetric (see [21]). However, it follows from the results in [17] that this case cannot occur when $\tau \neq 0$.

To finish the proof we still have to consider the case $\tau=0$. But then $\tau^{*}=0$ on $M$. In this case it follows from (2.50) that $M$ is again a 2-stein space and so the result follows from [21].

\section{REFERENCES}

[1] J. Berndt, F. Prüfer and L. Vanhecke, Symmetric-like Riemannian manifolds and geodesic symmetries, Proc. Roy. Soc. Edinburgh Sect. A, 125 (1995), 265-282.

[2] J. Berndt, F. Tricerri and L. Vanhecke, Generalized Heisenberg groups and Damek-Ricci harmonic spaces, Lecture Notes in Math., 1598, Springer-Verlag, Berlin, Heidelberg, New York, 1995.

[3] A. L. Besse, Einstein manifolds, Ergeb. Math. Grenzgeb. 3. Folge 10, SpringerVerlag, Berlin, Heidelberg, New York, 1987.

[4] J. E. D'Atri, Geodesic spheres and symmetries in naturally reductive spaces, Michigan Math. J., 22 (1975), 71-76.

[5] J. E. D'Atri, H. K. Nickerson, Divergence-preserving geodesic symmetries, J. Differential Geom., 3 (1969), 467-476.

[6] J. E. D'Atri, H. K. Nickerson, Geodesic symmetries in spaces with special curvature tensors, J. Differential Geom., 9 (1974), 251-262.

[7] G. Grantcharov and O. Muškarov, Hermitian *-Einstein surfaces, Proc. Amer. Math. Soc., 120 (1994), 233-239..

[8] A. Gray and L. Vanhecke, Riemannian geometry as determined by the volumes of small geodesic balls, Acta Math., 142 (1979), 157-198.

[9] P. Günther and F. Prüfer, D'Atri spaces are ball-homogeneous, preprint 1995.

[10] J. L. Kazdan, personal communication.

[11] T. Koda, A remark on the manifold $\mathbb{C P}^{2} \# \overline{\mathbb{C P}^{2}}$ with Bérard-Bergery's metric, Ann. Global Anal. Geom., 11 (1993), 323-329.

[12] O. Kowalski, Spaces with volume-preserving symmetries and related classes of Riemannian manifolds, Rend. Sem. Mat. Univ. Politec Torino, Fascicolo Speciale Settembre (1983), 131-158.

[13] O. Kowalski, F. Prüfer and L. Vanhecke, D'Atri spaces, Topics in Geometry: In Memory of Joseph D'Atri (S. Gindikin, ed.), Progress in Nonlinear Diff. Eq., 20 (1996), Birkhäuser, Boston, Basel, Berlin, 241-284. 
[14] C. Le Brun, Einstein metrics on complex surfaces, preprint 1995.

[15] D. Page, A compact rotating gravitational instanton, Phys. Lett., 79B (1979), 235-238.

[16] F. Prüfer, On compact Riemannian manifolds with volume-preserving symmetries, Ann. Global Anal. Geom., 7 (1989), 133-140.

[17] K. Sekigawa, On 4-dimensional connected Einstein spaces satisfying the condition $R(X, Y) \cdot R=0$, Sem. Rep. Niigata Univ., A7 (1969), 29-31.

[18] K. Sekigawa, On some 4-dimensional compact almost Hermitian manifolds, J. Ramanujan Math. Soc., 2 (1987), 101-116.

[19] K. Sekigawa and L. Vanhecke, Symplectic geodesic symmetries on Kähler manifolds, Quart. J. Math. Oxford (2), 37 (1986), 95-103.

[20] K. Sekigawa and L. Vanhecke, Volume-preserving geodesic symmetries on fourdimensional Kähler manifolds, Differential Geometry Peñiscola 1985, Lecture Notes in Math., 1209, Springer-Verlag, Berlin, Heidelberg, New York (1986), pp. 275-291.

[21] K. Sekigawa and L. Vanhecke, Volume-preserving geodesic symmetries on fourdimensional 2-stein spaces, Kodai Math. J., 9 (1986), 215-224.

[22] Z. I. Szabó, Spectral theory for operator families on Riemannian manifolds, Proc. Sympos. Pure Math., 54 (1993), 615-665.

[23] F. Tricerri and I. Vaisman, On some 2-dimensional Hermitian manifolds, Math. Z., 192 (1986), 205-216.

[24] F. Tricerri and L. Vanhecke, Curvature tensors on almost Hermitian manifolds, Trans. Amer. Math. Soc., 267 (1981), 365-398.

[25] I. Vaisman, Non-Kähler metrics on geometric complex surfaces, Rend. Sem. Math. Univ. Politec Torino, 45 (1987), 117-123.

[26] L. Vanhecke, Geometry in normal and tubular neighborhoods, Proc. Workshop on Differential Geometry and Topology Cala Gonone (Sardinia) 1988, Rend. Sem. Fac. Sci. Univ. Cagliari, Supplemento al vol. 58 (1988), 73-176.

[27] L. Vanhecke and T. J. Willmore, Interaction of tubes and spheres, Math. Ann., 263 (1983), 31-42.

[28] T. J. Willmore, Riemannian geometry, Oxford Science Publications, Clarendon Press, Oxford, 1993.

\author{
J. T. Cho \\ Topology and Geometry Research Center \\ Kyungpook National University \\ Taegu 702-701, \\ Korea \\ K. Sekigawa \\ Department of Mathematics \\ Niigata University \\ Niigata 950-21, \\ Japan
}


L. Vanhecke

Department of Mathematics

Katholieke Universiteit Leuven

Celestijnenlaan 200B, 3001 Leuven,

Belgium 